\title{
GC Content of the Rumen Fungal DNA
}

\author{
V Kostyukovsky 1, C Kohda 2, Y Nakai 2, T Ando 2, T Inamoto ${ }^{2}, \mathrm{~K}$ Ogimoto ${ }^{2}$ \\ Laboratory of Anaerobic Processes, Institute of Biochemistry and Physiology of Microorganisms, Puschino, \\ Moscow Region, 142292, Russia ; 2Department of Animal Microbiology and Parasitology, Tohoku University, 1-1 \\ Tsutsumi-dori, Amamiya-cho, Aoba-ku, Sendai 981, Japan
}

Taxonomic relations between rumen fungi and other Chytridiomycetes remain uncertain. Guanine plus cytosine (GC) content of the DNA is considered to be an important criterion in microbial taxonomy. Characteristics of the DNA from several rumen fungi have been described (Brownlee, 1989a, NAR, 17, 13271335 ; Billon-Grand et al, 1991, FEMS Microbiol Lett, 82, 267-270 ; Fiol et al, 1992, Ann Zootech, 41, 77-78). We determined GC content of the DNA from 5 more rumen anaerobic Chytridiomycetes.

Strains Neocallimastix sp. TU1 from ovine and Neocallimastix sp. TU2, Piromyces sp. TU3, unidentified polycentric fungus TU4 and Caecomyces sp. TU5 from bovine rumen were isolated by the method described earlier (Kostyukovsky et al, 1991, J General Microbiol, 137, 1759-1764).

DNA was extracted as the method of Raeder and Broda (1985, Lett Appl Microbiol, 1, 17$20)$. The method allowed us to get sufficient amount of good purity DNA in less than $2 \mathrm{~h}$. The total bulk of the DNA was then degraded enzymatically (Mesbah et al, 1989, Int J Systematic Bacteriol, 39, 159-167) and subjected to the HPLC analysis. As it was noted earlier (Billon-Grand et al, 1991) when dealing with total DNA, HPLC technique gives average content for the genome.
The DNA showed significant differences in GC content (Table), the latter being also considerably higher than those reported earlier (Brownlee, 1989a ; Billon-Grand et al, 1991, Fiol et al, 1992). This might result either from (i) a contamination with mycoplasmas which are often associated with rumen fungi (Kudo et al, 1990, Can J Microbiol, 36, 513-517) or (ii) the DNA isolation procedure. (i) The purity of our strains had been revealed by microscopy of roll-tube cultures, and we believe that the strains studied by the other researchers were not contaminated as well. (ii) We tried to isolate the DNA in as little steps as possible. Though fast, the method might cause losses of the DNA owing to the degradation of more liable AT-rich part of genome. We also did not segregate any fractions of the DNA as it was done by the above mentioned authors, who separated AT-rich DNA from the more GC-rich satellite DNA.

More data on a rumen fungal DNA specificities from other laboratories in different geographical locations are necessary. Alternatively, the DNA probe specific for the fungal genera (Brownlee, 1989b, In : The roles of protozoa and fungi in ruminant digestion (JV Nolan, RA Leng, DI Demeyer, eds), Penambul Books : Armidale, Australia, 251-253) may prove to be more promising in taxonomy of anaerobic Chytridiomycetes.

\begin{tabular}{lccc}
\hline Strains & DNA yield, \% DM & $\mathrm{OD}_{260} / \mathrm{OD}_{280}$ & $\mathrm{GC}$ content, \% \\
\hline Neocallimastix sp. TU1 & 0.16 & 1.93 & 50.50 \\
Neocallimastix sp. TU2 & 0.28 & 1.97 & 32.70 \\
Piromyces sp. TU3 & 0.67 & 2.12 & 52.48 \\
Unidentified strain TU4 & 0.47 & 1.94 & 48.38 \\
Caecomyces sp. TU5 & 0.07 & 1.94 & 49.64
\end{tabular}

